\title{
Interpretation of bronchograms and chest radiographs in patients with chronic sputum production
}

\author{
D C CURRIE, J C COOKE, A D MORGAN, I H KERR, D DELANY, \\ B STRICKLAND, P J COLE
}

From the Host Defence Unit, Department of Thoracic Medicine, Cardiothoracic Institute, and Department of Radiology, Brompton Hospital, London

ABSTRACT Bronchograms and plain chest radiographs of 27 patients with chronic sputum production were reported separately in random order and independently by two pulmonary radiologists to establish the diagnostic rate of each investigation and to assess interobserver variation.

Both radiologists agreed on the presence of bronchiectasis on bronchography in 19 of $27(70 \%)$ patients and in 94 of $448(21 \%)$ bronchopulmonary segments. One radiologist only interpreted the films as showing bronchiectasis in a further two (7\%) patients and $26(6 \%)$ segments. There was more disagreement about the presence or absence of individual bronchographic abnormalities.

Two main groups of patients with bronchiectasis were identified by bronchography: 11 with bronchiectasis alone and eight with bronchiectasis and bronchographic features suggestive of "chronic bronchitis". There was no clinical difference between these two groups.

Plain chest radiographs were insensitive, being diagnostic (both radiologists agreeing) of bronchiectasis in only nine of $19(47 \%)$ patients with definite bronchiectasis on bronchography.

Bronchiectasis is usually defined as irreversible dilatation of the bronchial tree. Reid described three different bronchographic appearances of bronchiectasis-namely, cylindrical, varicose, and saccular (cystic) - and related these to pathological changes in the bronchi. ${ }^{1}$ In cylindrical bronchiectasis the bronchi have regular outlines, show no great increase in diameter, and usually end squarely and abruptly. Varicose bronchi show irregular dilatation and bulbous termination, while saccular bronchiectasis is characterised by bronchial dilatation that increases progressively towards the periphery of the lung, with a ballooned outline and few bronchial subdivisions.

By contrast, in established chronic bronchitis (cough, sputum, and breathlessness for more than one year with some degree of disability without evidence of another cause) $)^{2}$ the principal bronchographic abnormalities are localised beaded bronchial dilatation, diverticulosis (mucus pits), poor peripheral filling of bronchi and bronchioles, peripheral pooling of contrast medium, and excessive vari-

\footnotetext{
Address for reprint requests: Dr P J Cole, Host Defence Unit, Department of Thoracic Medicine, Cardiothoracic Institute, Fulham Road, London SW36HP.
}

Accepted 1 October 1986 ation of bronchial calibre on respiration. ${ }^{3}$ In early chronic bronchitis (mucus hypersecretion without breathlessness) the reported abnormalities are minor irregularities of the calibre of medium sized bronchi, mucus ducts (pits), and incomplete delineation of the distal parts of the bronchial tree. ${ }^{4}$

Dilatation of the bronchial tree is an important feature of both bronchiectasis and chronic bronchitis, so we decided to study the bronchograms of patients with chronic sputum production to investigate difficulties in diagnosis and assess the importance of these abnormalities.

\section{Patients and methods}

\section{PATIENTS}

Twenty seven consecutive patients with chronic sputum production, referred for bronchography to try to establish the cause of their sputum production, were studied. Their mean age was 42 years (range 18-65 years). There were 14 women and 13 men. Twenty six had expectorated sputum every day for a median duration of eight years (range 0.8-49 years), with purulent sputum in 24; one expectorated sputum intermittently. Haemoptysis had occurred in eight patients. The patients had a mean forced expiratory volume in one second $\left(\mathrm{FEV}_{1}\right)$ of $84 \%$ predicted 
(range 40-117\%). Two were smokers and seven exsmokers. Fifteen patients reported nasal symptoms, two had rheumatoid arthritis, one had Crohn's disease, one had allergic bronchopulmonary aspergillosis, and one panhypogammaglobulinaemia; eight patients reported a history of pneumonia, five of whooping cough, and one each of measles and tuberculosis.

\section{IN VESTIGATIONS}

Twenty seven bronchograms and standard posteroanterior chest radiographs and 20 lateral plain chest radiographs were performed. Twenty three bronchograms were bilateral and the remainder unilateral-that is, 50 lungs. Bronchograms were performed between acute exacerbations of chest symptoms in all but one patient. Chest physiotherapy preceded bronchography whenever necessary and was performed routinely afterwards. The contrast medium, an aqueous suspension of propyliodone (Dionosil, Glaxo), was introduced by fibreoptic bronchoscope in 15 patients and through a nasal catheter in 12 . Ten to $20 \mathrm{ml}$ of contrast were instilled into each lung to achieve adequate filling of the bronchial tree. A total of 448 bronchopulmonary segments were examined.

\section{ANALYSIS}

Two pulmonary radiologists independently reported on each chest radiograph and bronchogram on separate occasions, unaware of the patient's identity. The only clinical information available to the radiologists was that the patient was being investigated for sputum production. Standard report forms were designed to record specific abnormalities and a diagnostic interpretation at segmental level on the bronchogram and in zones on the radiograph.

The diagnostic interpretation was subjective, based on the experience of each radiologist, and was not directly calculated from the presence or absence of specific abnormalities. On the radiographs each lung was divided into upper, middle, and lower zones. The boundary between the upper and middle zone was defined as a horizontal line drawn at the level of the anterior end of the second rib and that between middle and lower zone as a horizontal line drawn at the level of the anterior end of the fourth rib. In 20 patients with lateral films as well as posteroanterior chest radiographs diagnostic interpretation on plain radiography was also assessed at lobar level. Accurate and complete form filling was ensured by another author, who attended all the reporting sessions. The bronchographic features assessed were proximal dilatation, distal dilatation, non-tapering of bronchi, lack of bronchial side branches, and luminal filling defects. The presence or absence of mucus pits was also recorded for each bronchogram. The radiograph was assessed for the presence of linear markings, "bronchial wall thickening", patchy or confluent pulmonary shadowing, pleural thickening, evidence of lung collapse or volume loss, and circular markings; these features were derived from the observations of Güdjberg. ${ }^{5}$

"Bronchographic chronic bronchitis" was defined as a combination of bronchographic features regarded by the radiologists as highly suggestive of the diagnosis of clinical chronic bronchitis. The features regarded as important were localised beaded bronchial dilatation, minor irregularities of calibre of medium sized bronchi, mucus pits, incomplete delineation of the distal parts of the bronchial tree, and peripheral pooling of contrast medium.

A third pulmonary radiologist provided a diagnostic interpretation (though not specific features) on all radiographs. These were consulted when there was disagreement between the first two radiologists.

\section{Results}

\section{BRONCHOGRAMS}

Table 1 shows the frequency of reporting of the five bronchographic features in the 448 segments. Agreement and disagreement between the two radiologists is indicated in separate columns. Table 2 shows the frequency of the diagnosis of bronchiectasis in segments, lobes, and lungs and in patients. There was considerably less disagreement between the two radiologists about the diagnosis than about the presence

Table 1 Analysis of bronchographic features in 448 bronchopulmonary segments. Values are No ( $\%$ of total segments examined)

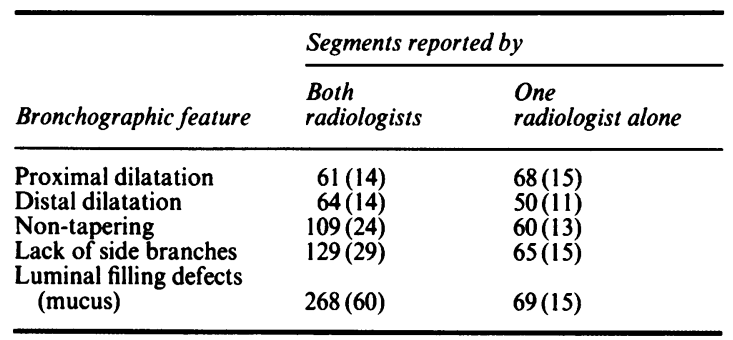

Table 2 Diagnosis of bronchiectasis from bronchograms. Values are No ( $\%$ of total examined)

\begin{tabular}{|c|c|c|c|}
\hline \multirow[b]{2}{*}{$\begin{array}{l}\text { Level of } \\
\text { interpretation }\end{array}$} & \multirow[b]{2}{*}{$n=$} & \multicolumn{2}{|c|}{ Diagnosis made by } \\
\hline & & $\begin{array}{l}\text { Both } \\
\text { radiologists }\end{array}$ & $\begin{array}{l}\text { One radiologist } \\
\text { only }\end{array}$ \\
\hline $\begin{array}{l}\text { Segment } \\
\text { Lobe } \\
\text { Lung } \\
\text { Patient }\end{array}$ & $\begin{array}{r}448 \\
126 \\
50 \\
27\end{array}$ & $\begin{array}{l}94(21) \\
43(34) \\
29(58) \\
19(70)\end{array}$ & $\begin{array}{c}26(6) \\
12(10) \\
3(6) \\
2(7)\end{array}$ \\
\hline
\end{tabular}


Table 3 Frequency of individual bronchographic features in segments with definite bronchiectasis and in other segments. Values are \% of total No of segments with diagnosis showing feature (No of segments)

\begin{tabular}{|c|c|c|}
\hline \multirow[b]{2}{*}{ Bronchographic feature } & \multicolumn{2}{|l|}{ Diagnosis } \\
\hline & $\begin{array}{l}\text { Bronchiectasis* } \\
\text { (94 segments) }\end{array}$ & $\begin{array}{l}\text { Other } \\
\text { (354 segments) }\end{array}$ \\
\hline $\begin{array}{l}\text { Proximal dilatation } \\
\text { Distal dilatation } \\
\text { Non-tapering } \\
\text { Lack of side branches } \\
\text { Mucus } \\
\text { Any combination of Proximal }\end{array}$ & $\begin{array}{l}55(52) \\
68(64) \\
91(86) \\
93(87) \\
93(87)\end{array}$ & $\begin{array}{l}3(9) \\
0(0) \\
6(23) \\
12(42) \\
51(181)\end{array}$ \\
\hline and Non-tapering & $98(92)$ & $8(30)$ \\
\hline
\end{tabular}

*Agreed as present by the first two radiologists.

or absence of individual abnormalities. Table 3 shows the relative value of the different bronchographic features for predicting bronchiectasis.

The first two radiologists concluded that 19 of the 50 lungs studied had definite bronchiectasis alone, 10 lungs had definite bronchiectasis with bronchographic chronic bronchitis, 11 had bronchographic chronic bronchitis alone, and five had features of excess mucus only. Five lungs were regarded as bronchographically normal.

The two radiologists agreed that of the 27 patients, 11 had bronchiectasis alone, eight had bronchiectasis with bronchographic chronic bronchitis, four had bronchographic chronic bronchitis alone, and four had no abnormality, apart from mucus in one. Only one patient had saccular bronchiectasis. Mucus pits were seen in patients from all diagnostic categories.

Of the 26 segments reported as bronchiectatic by only one radiologist, the other radiologist reported bronchographic chronic bronchitis in eight segments, non-diagnostic abnormal features in six, mucus alone in seven, underfilling of the bronchial tree in two, and a normal appearance in three. When the opinion of the third radiologist was included the number of segments diagnosed as definite bronchiectasis (reported as such by two or three of the radiologists) only increased by a further four segments to $98(21 \%)$ of

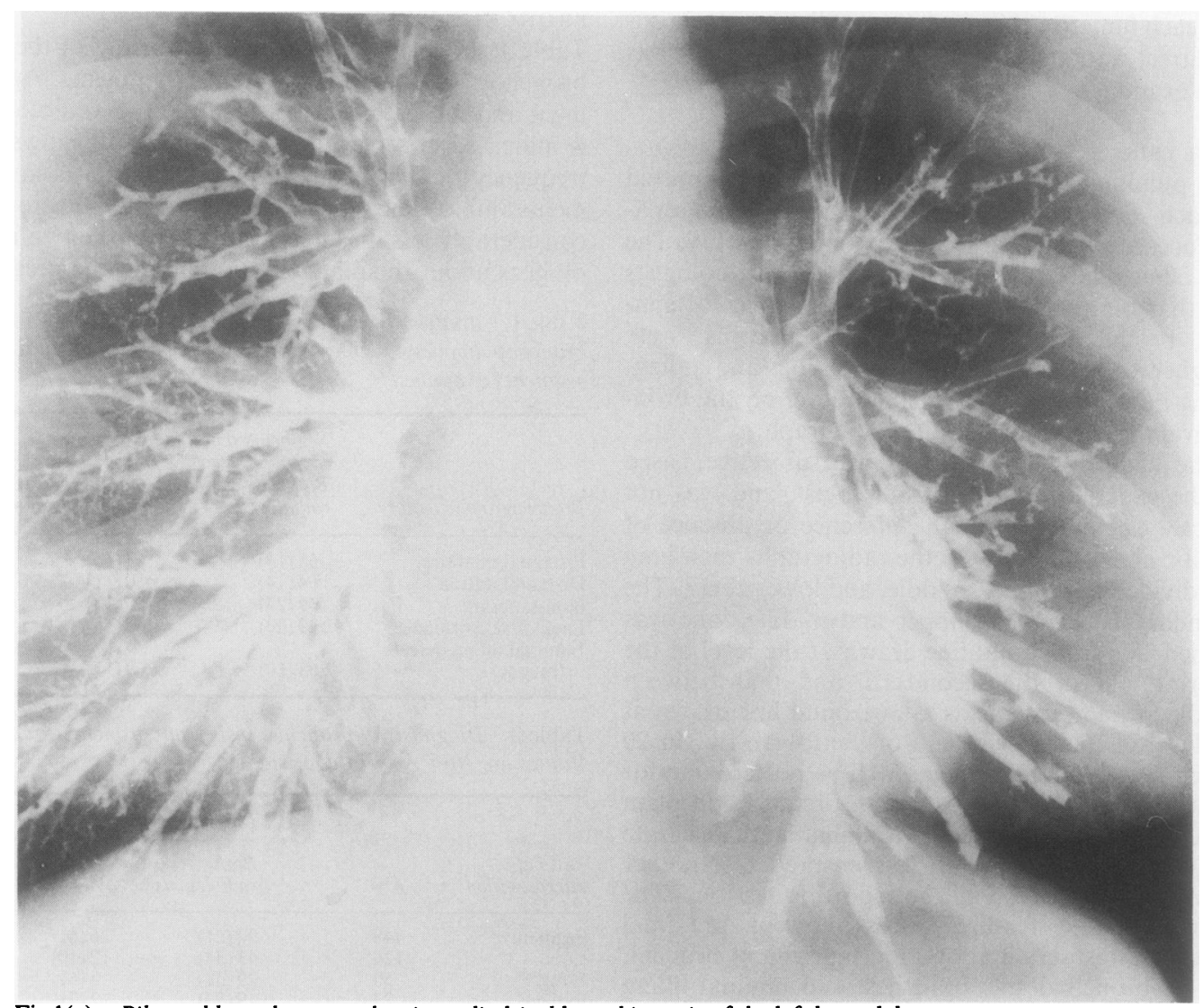

Fig 1(a) Bilateral bronchogram, showing cylindrical bronchiectasis of the left lower lobe. 
the 448 segments.

Figures $1(\mathrm{a})$ and (b) show definite lobar bronchiectasis, figure 1(c) illustrates a technical problem, and figure 2 shows a patient with both bronchiectasis and radiographic chronic bronchitis.

There was no association between the presence or absence of bronchographic chronic bronchitis or bronchiectasis and the clinical characteristics of the patients (age, history, $\mathrm{FEV}_{1}$, smoking history, or associated syndromes).

\section{CHEST RADIOGRAPHS}

Table 4 shows the frequency of reporting of the six chest radiographic features studied in each of 147 zones. One radiograph was not available for analysis. There was considerable disagreement over the presence or absence of individual features. The radiograph showed definite bronchiectasis (with both radiologists agreeing) in nine (47\%) of the 19 patients who had definite bronchiectasis on bronchography (no false positives). Only two further patients were

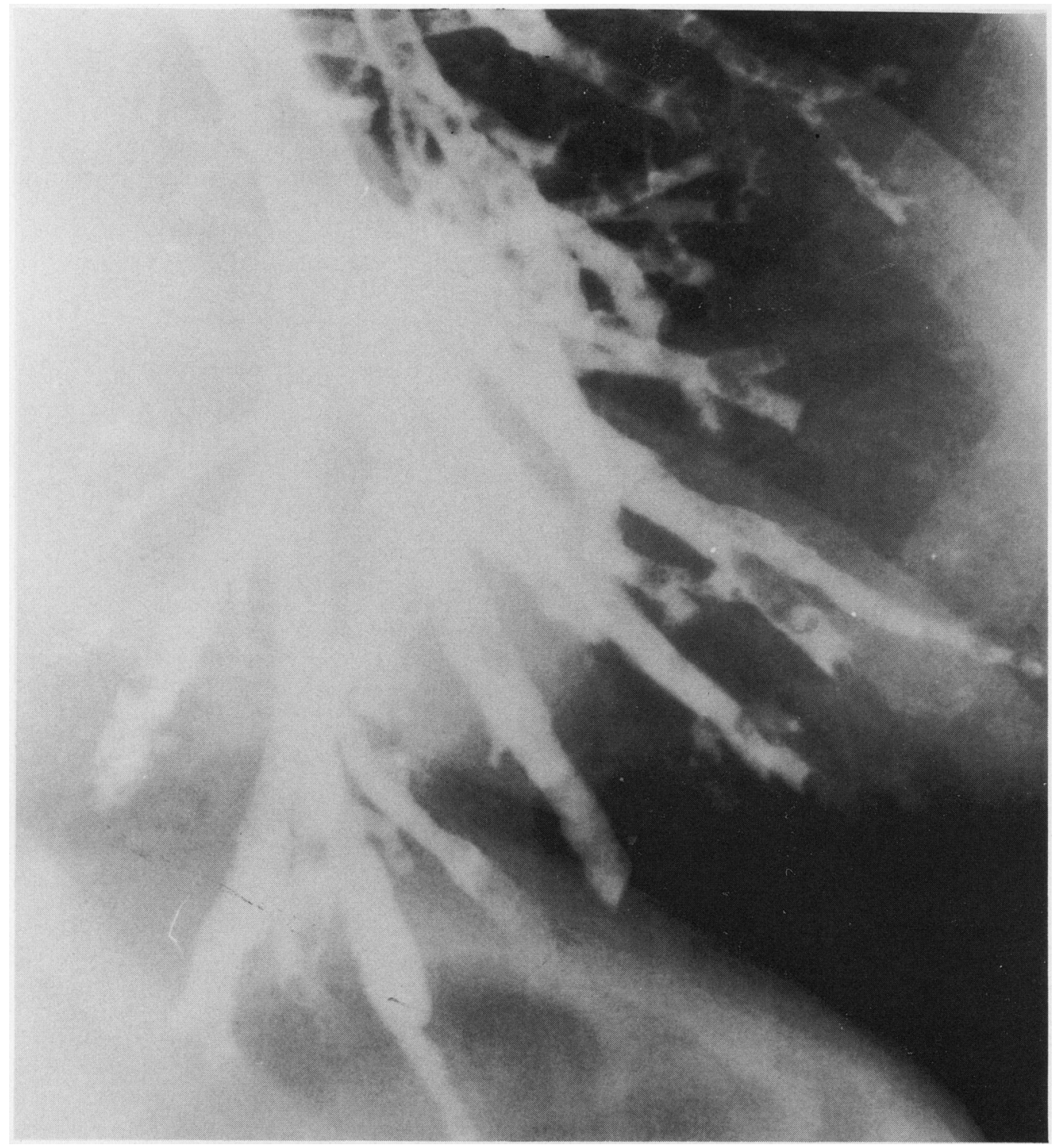

Fig 1(b) Close up of bronchogram in figure 1(a), showing the left lower lobe with proximal and distal dilatation, non-tapering, lack of side branches, and luminal filling defects (mucus). 
Currie, Cooke, Morgan, Kerr, Delany, Strickland, Cole $\underset{.}{\stackrel{3}{\vec{D}}}$

Table 4 Analysis of features on ptain posteroanterior chest radiographs in 147 zones. Values are No (\% of 147 zones)

\begin{tabular}{lll}
\hline & \multicolumn{2}{l}{ Zones reported by } \\
\cline { 2 - 3 } Feature & $\begin{array}{l}\text { Both } \\
\text { radiologists }\end{array}$ & $\begin{array}{l}\text { One } \\
\text { radiologist alone }\end{array}$ \\
\hline Linear markings & $16(11)$ & $27(18)$ \\
Bronchial wall thickening & $20(14)$ & $21(14)$ \\
$\begin{array}{l}\text { Confluent/patchy } \\
\text { shadowing }\end{array}$ & $7(5)$ & $7(15)$ \\
Collapse/loss of volume & $4(3)$ & $8(5)$ \\
Pleural thickening & $6(4)$ & $5(3)$ \\
Circular markings & $0(0)$ & $1(1)$ \\
\hline
\end{tabular}

One chest radiograph could not be traced for analysis.

considered to have bronchiectasis on radiography by one radiologist. If analysed at lung level radiography had a sensitivity of $37 \%$ compared with bronchography (11 of 30 lungs with definite bronchiectasis on bronchography) for the diagnosis of bronchi- $\overrightarrow{\vec{O}}$ ectasis, with a false positive rate of $5 \%$ (one of 19 lungs). A further three lungs were considered to have $\frac{\bar{\sigma}}{\sigma}$ bronchiectasis on radiography by one radiologist.

The sensitivity for the diagnosis of bronchiectasis on plain chest radiography compared with bronchography was no better when both lateral and posteroanterior views were assessed. The sensitivity at $\vec{\circ}$ lobar level in these 20 patients (113 lobes) was $13 \% \overrightarrow{\overrightarrow{\vec{t}}}$ (five of 38 lobes with definite bronchiectasis on bronchography), with a false positive rate of $5 \%$ (four of $\vec{x}$ 75 lobes).

\section{Discussion}

Bronchiectasis was common in this group of patients with chronic sputum production. Although the patients were consecutively enrolled, the fact that

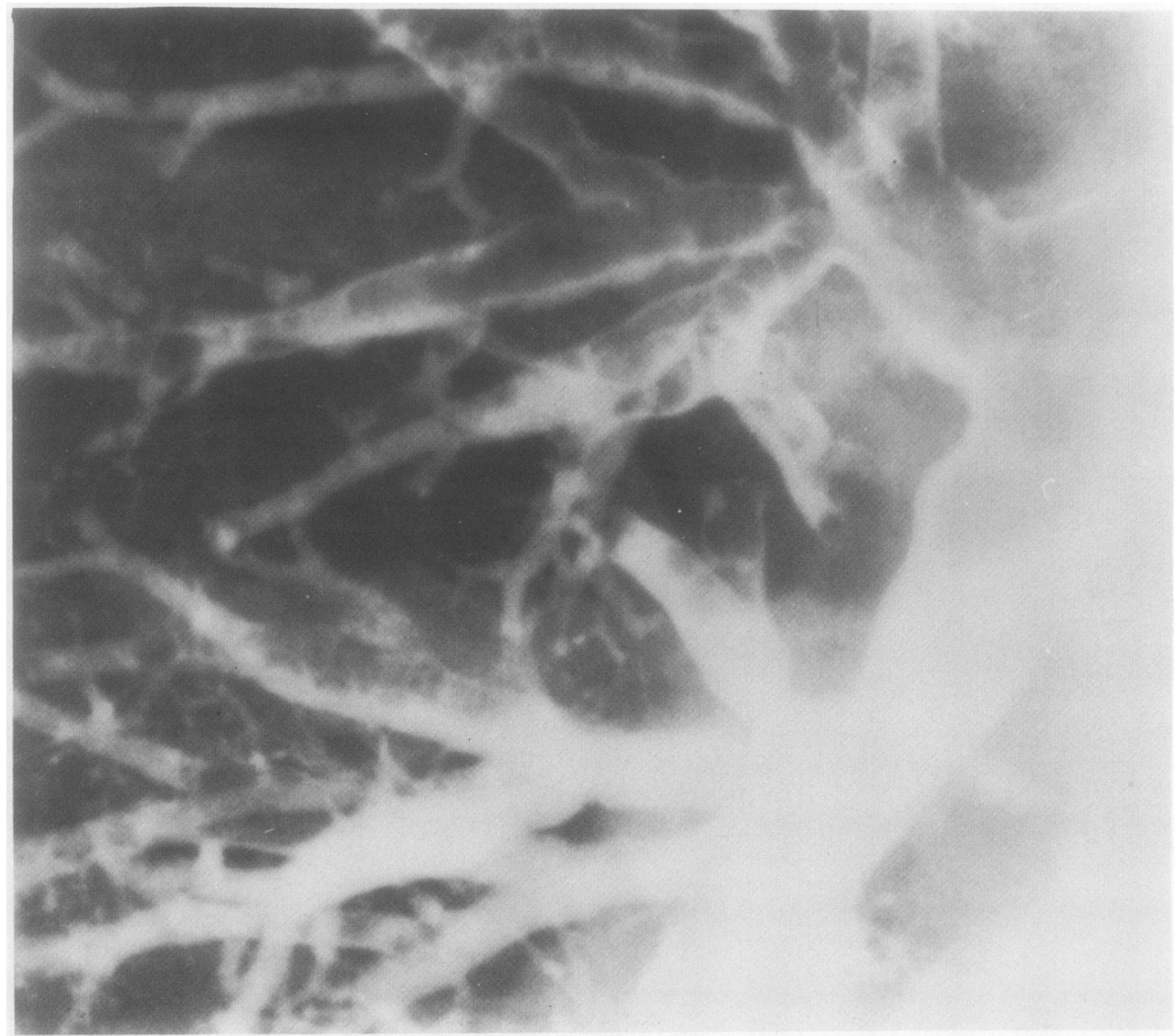

Fig 1(c) Close up of the apical segment of the right lower lobe in figure 1(a), seeming to show proximal dilatation and bronchiectasis. The bronchial tree may in fact be normal, seeming to be proximally dilated as a result of poor filling with contrast medium. 
they were referred for bronchography to investigate their symptoms tended to select those with a history suggestive of bronchiectasis and those with an abnormal plain chest radiograph. Plain chest radiography was diagnostic of bronchiectasis in only $47 \%$ of the patients with bronchographically diagnosed bronchiectasis, and bronchiectasis was mistakenly diagnosed in one lung reported as "chronic bronchitis" on bronchography. The poor sensitivity of plain posteroanterior chest radiography makes it unacceptable for assessment of the extent of bronchiectasis before

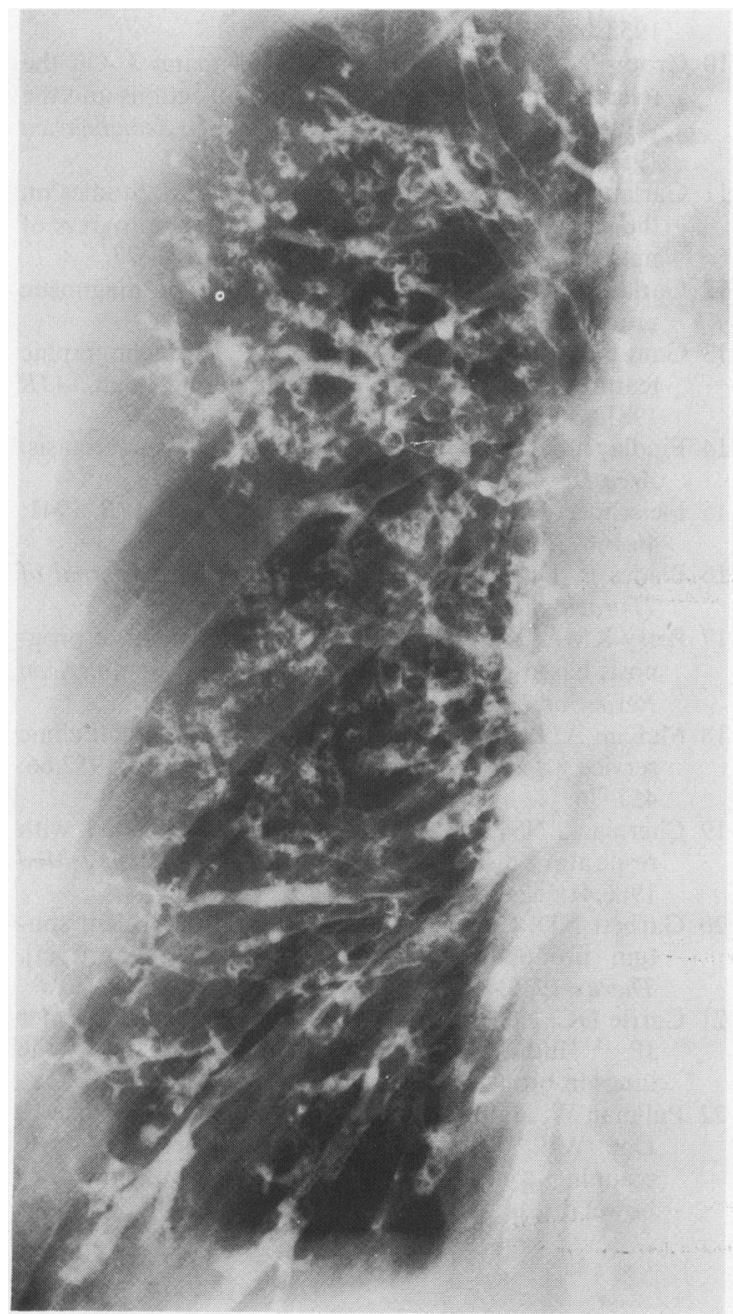

Fig 2 Bronchogram of the right lung of another patient, showing a range of bronchial abnormalities from definite bronchiectasis in segments of the lower lobe to bronchographic chronic bronchitis characterised by localised beaded bronchial dilatation, minor irregularities of the calibre of medium sized bronchi, incomplete delineation of distal parts of the bronchial tree, and peripheral pooling of contrast medium in the uppper lobe. surgery. Assessment of the lateral as well as the posteroanterior view did not improve the sensitivity of plain chest radiography.

The two radiologists disagreed about the diagnosis of bronchiectasis in $22 \%$ ( 26 of 120) of all segments regarded as bronchiectatic by one or both radiologists. This level of disagreement is similar to that seen in studies that have assessed the reliability of reading of chest radiographs for the presence of tuberculosis. ${ }^{6-12}$ Disagreement over the presence of specific bronchographic features was greater. This was most pronounced for proximal dilatation, with disagreement in $53 \%$ (68 of 129) of segments regarded as positive by one or both radiologists. The level of disagreement for the presence of distal dilatation was $44 \%$ ( 50 of 114 ), for non-tapering was $36 \%$ (60 of 119 ), and for lack of side branches was $34 \%$ (65 of 194). The disagreement between two experienced pulmonary radiologists over the diagnosis and presence or absence of individual bronchographic features highlights the difficulty of interpreting bronchograms. Our radiologists were asked to make a diagnostic interpretation based on experience rather than on a scoring system determined by the presence or absence of particular features. Reassuringly, the presence of any combination of proximal dilatation, distal dilatation, and non-tapering agreed by both radiologists would have diagnosed $98 \%$ of the bronchopulmonary segments diagnosed as bronchiectatic by both radiologists, with a false positive rate of $8 \%$.

The patients could be separated into four groups on the basis of the results of their bronchograms: those with bronchiectasis alone, those with bronchiectasis with bronchographic chronic bronchitis, those with bronchographic chronic bronchitis alone, and those with no important bronchographic abnormality.There were no clinical differences between these groups, particularly with regard to the presence of smokers. The importance of bronchographic chronic bronchitis in terms of the cause, symptoms, and natural history of the condition is unknown and bronchographic features suggestive of clinical chronic bronchitis have been reported recently in 13 apparently healthy subjects. ${ }^{13}$ Chronic bronchitis may be defined clinically, pathologically, and bronchographically, and patients diagnosed on the basis of one definition may not meet the criteria of another definition. An additional complication is that bronchial dilatation on bronchography of similar degree to that seen with bronchiectasis, and occurring within one year of an episode of pneumonia, may disappear spontaneously. ${ }^{14-16}$ The importance of differences in the degree of bronchial dilatation and the bronchial contour is debated. Two studies before the era of antibiotics reported that patients with saccular disease had a worse prognosis than patients 
with cylindrical disease. ${ }^{1718} \mathrm{~A}$ major study since the advent of antibiotics noted, however, that patients with saccular and cylindrical disease of similar distribution had similar lung function. ${ }^{19}$

In view of our results we urge caution in the interpretation of bronchograms in patients with chronic sputum production. Abnormalities interpreted as bronchiectasis by one radiologist may not be interpreted as such by another. Clinically, we have seen patients with daily purulent sputum production without bronchographic evidence of bronchiectasis ${ }^{20}$ and, conversely, patients with mucoid sputum and definite bronchiectasis. We advocate that all such patients are medically managed as if they have bronchiectasis unless evidence is forthcoming that bronchographic differences are important.

Bronchography remains the best established method of imaging the macroscopic anatomy of the bronchial tree and is still the investigation of choice to ascertain the extent of disease in patients with chronic sputum production when surgery is being considered for apparently localised disease. Ideally, however, identification of actively inflamed parts of the bronchial tree, which may be the source of abnormal mucus production, is required. These inflamed areas need not be confined to bronchographically abnormal bronchi, and, conversely, some bronchiectatic bronchi may be quiescent. A different method of imaging - for example, ${ }^{11}$ indium or ${ }^{99} \mathrm{~m}$ technetium labelled leucocyte scanning ${ }^{2122}$-is required to delineate actively inflamed bronchi.

DC was supported by the Chest, Heart and Stroke Association.

\section{References}

1 Reid LM. Reduction in bronchial subdivisions in bronchiectasis. Thorax 1950;5:233-47.

2 Oswald NC, Harold JT, Martin WJ. Clinical patterns of chronic bronchitis. Lancet 1953;ii:639-43.

3 Simon G, Galbraith HJB. Radiology of chronic bronchitis. Lancet 1953;ii:850-2.

4 Gregg I, Trapnell DH. The bronchographic appearances of early chronic bronchitis. Br J Radiol 1969;42:132-9.

5 Güdjberg CE. Roentgenologic diagnosis of bronchi-
6 Birkelo CC, Chamberlain WE, Phelps PS, Schools PE Zacks D, Yerushalmy J. Tuberculosis case finding: a comparison of the effectiveness of various roent genographic and photofluorographic methods. JAM 1947;133:359-66.

7 Garland LH. On the scientific evaluation of diagnosti@e procedures. Radiology 1949;52:309-27.

8 Yerushalmy J, Harkness JT, Cope JH, Kennedy BR. The $\vec{\circ}$ role of dual reading in mass radiography. American $\vec{Z}$ Review of Tuberculosis 1950;61:443-64.

9 Garland LH, Cochrane AL. Results of an internationalf test in chest roentgenogram interpretation. $J A M \mathbb{A}^{\times}$ 1952;149:631-4.

10 Groth-Peterson E, Løvgreen A, Thillemann J. On the reliability of the reading of photofluorograms and the value of dual reading. Acta Tuberculosea Scandinavica 1952;26:13-37.

11 Garland LH, Miller ER, Zwerling HB, et al. Studies onthe value of serial films in estimating the progress of pulmonary disease. Radiology 1952;58:161-77.

12 Garland LH. Studies on the accuracy of diagnostic. procedures. AJR 1959;82:25-38.

13 Gamsu G, Forbes AR, Ovenfors C. Bronchographic features of chronic bronchitis in normal men. $A J R$ 1981;136:317-22.

14 Findlay L. Atelectatic or compensatory bronchiectasis? Arch Dis Child 1935;10:61-84.

15 Fleischner FG. Reversible bronchiectasis. AJR $1941 \stackrel{2}{\frac{2}{2}}$ 46:166-72.

16 Blades B, Dugan DJ. Pseudobronchiectasis. Journal os Thoracic Surgery 1944;13:40-8.

17 Perry KMA, King DS. Bronchiectasis: a study of prog $\underset{P}{\vec{P}}$ nosis based on a follow up of $\mathbf{4 0 0}$ patients. American? Review of Tuberculosis 1940;41:531-47.

$18 \mathrm{McKim}$ A. Bronchiectasis as seen in an ambulant clinie service. American Review of Tuberculosis 1952;6600 457-76.

19 Cherniack NS, Carton RW. Factors associated with respiratory insufficiency in bronchiectasis. Am J Me 1966;41:562-71.

20 Garbett ND, Currie DC, Cole PJ. Daily purulent spu tum production without bronchiectasis (abstract $\mathrm{B}$ Thorax 1986;41:720.

21 Currie DC, Needham S, Peters AM, Cole PJ, Lavende? JP. ${ }^{111}$ Indium labelled neutrophils migrate to th lungs in bronchiectasis. Thorax 1986;41:256.

22 Pullman W, Hanna R, Sullivan P, Booth JA, Lomas FN Doe WF. Technetium-99m autologous phagocyte scanning: a new imaging technique for inflammator bowel disease. $\mathrm{Br}$ Med J 1986;293:161-4. 